\title{
Association between somatosensory evoked potentials and EEG in comatose patients after cardiac arrest
}

\author{
Astrid B. Glimmerveen ${ }^{\mathrm{a}, *}$, Barry J. Ruijter ${ }^{\mathrm{b}}$, Hanneke M. Keijzer ${ }^{\mathrm{a}, \mathrm{c}}$, Marleen C. Tjepkema-Cloostermans ${ }^{\mathrm{b}, \mathrm{d}}$, \\ Michel J.A.M. van Putten ${ }^{\mathrm{b}, \mathrm{d}}$, Jeannette Hofmeijer ${ }^{\mathrm{a}, \mathrm{b}}$ \\ a Department of Neurology, Rijnstate Hospital, P.O. Box 9555, 6800 TA Arnhem, The Netherlands \\ ${ }^{\mathrm{b}}$ Clinical Neurophysiology, Technical Medical Centre, University of Twente, P.O. Box 217, 7500 AE Enschede, The Netherlands

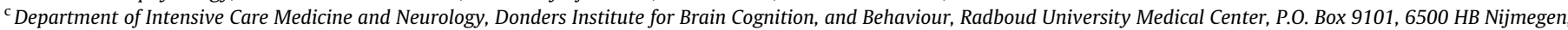 \\ The Netherlands \\ ${ }^{\mathrm{d}}$ Department of Neurology and Clinical Neurophysiology, Medisch Spectrum Twente, P.O. Box 50000, 7500 KA Enschede, The Netherlands
}

\section{A R T I C L E I N F O}

\section{Article history:}

Accepted 18 August 2019

Available online 31 August 2019

\section{Keywords:}

EEG

SSEP

Prognosis

Postanoxic coma

Cardiac arrest

\section{H I G H L I G H T S}

- Absent SSEP and suppressed or synchronous EEG on suppressed background predict poor outcome.

- SSEP is either present or absent, with no changes over the first 5 days.

- SSEP cannot be predicted by EEG patterns, except for preserved N20 in normal EEG.

\section{A B S T R A C T}

Objective: To analyze the association between SSEP results and EEG results in comatose patients after cardiac arrest, including the added value of repeated SSEP measurements.

Methods: Continuous EEG was measured in 619 patients during the first 3-5 days after cardiac arrest. SSEPs were recorded daily in the first 55 patients, and on indication in later patients. EEGs were visually classified at 12, 24, 48, and $72 \mathrm{~h}$ after cardiac arrest, and at the time of SSEP. Outcome at $6 \mathrm{~m}$ was dichotomized as good (Cerebral Performance Category 1-2) or poor (CPC 3-5). SSEP and EEG results were related to outcome. Additionally, SSEP results were related to the EEG patterns at the time of SSEP. Results: Absent SSEP responses and suppressed or synchronous EEG on suppressed background $\geq 24 \mathrm{~h}$ after cardiac arrest were invariably associated with poor outcome. SSEP and EEG identified different patients with poor outcome (joint sensitivity $39 \%$ at specificity 100\%). N20 responses were always preserved in continuous traces at $>8 \mathrm{~Hz}$. Absent SSEPs did not re-emerge during the first five days. Conclusions: SSEP and EEG results may diverge after cardiac arrest.

Significance: SSEP and EEG together identify more patients without chance of recovery than one of these alone.

(c) 2019 International Federation of Clinical Neurophysiology. Published by Elsevier B.V. All rights reserved.

\section{Introduction}

Comatose patients after cardiac arrest have an uncertain prognosis. Despite treatment on intensive care units (ICUs), approximately half have a poor outcome as a result of severe postanoxic encephalopathy (Zandbergen et al., 1998). Early recognition of patients with and without chances of recovery of brain functioning

\footnotetext{
* Corresponding author. Fax: +31 880058890.

E-mail address: aglimmerveen@rijnstate.nl (A.B. Glimmerveen).
}

may prevent continuation of futile intensive care treatment and contribute to communication between doctors and families.

Somato-sensory evoked potential (SSEP) and EEG recordings hold valuable information for prediction of outcome. Absent SSEP responses after restoration of normothermia are strongly associated with a poor outcome and have been included in prognostication guidelines since the late 1990s (Nolan et al., 2015; Sandroni et al., 2014). Recent studies have shown the prognostic value of EEG patterns. An evolution towards continuous rhythms within twelve hours after arrest was strongly related to good outcome (Cloostermans et al., 2012; Hofmeijer et al., 2015; Oh et al. 2015; Sivaraju et al., 2015). Otherwise, lasting iso-electricity or 
synchronous patterns on a suppressed background invariably predicted a poor outcome (Cloostermans et al., 2012; Hofmeijer et al., 2015; Ruijter et al., 2019a; Sivaraju et al., 2015; Sondag et al., 2017; Spalletti et al., 2016).

Since previous authors have reported that EEG and SSEP are complementary for detection of patients with a poor outcome (Hofmeijer et al., 2015; Ruijter et al., 2019a), pathological EEG patterns and absent SSEPs probably reflect different manifestations of irreversible brain damage. In fact, EEG rhythms primarily reflect pyramidal cell functioning, including their synchrony, for which a sufficient number of functioning pyramidal synapses is required (Buzsaki et al., 2012). Otherwise, SSEP responses depend on intact thalamocortical projections (van Putten, 2012).

In the current study, we analyze the association between SSEP results and EEG results in comatose patients after cardiac arrest. We hypothesize that SSEP and EEG results may diverge and are complementary for reliable detection of patients with poor outcome. In addition, we analyze the added value of repeated SSEP measurements.

\section{Materials and methods}

\subsection{Study design}

This is a retrospective analysis of a prospective cohort study on continuous EEG registration of comatose patients after cardiac arrest, conducted on ICUs of two teaching hospitals in the Netherlands. In the Medisch Spectrum Twente (Enschede), patients were included from June 2010 to November 2017. In Rijnstate Hospital (Arnhem), patients were included from June 2012 to September 2017. Part of the EEG data were used in previous studies (Cloostermans et al., 2012; Hofmeijer et al., 2015, 2014; Ruijter et al., 2018, 2019a; Sondag et al., 2017; Tjepkema-Cloostermans et al., 2017, 2015).

\subsection{Standard protocol approvals, registrations, and patient consents}

EEG registration is part of current care in the two participating hospitals. The Medical Ethical Committee Twente approved the protocol and waived the need for informed consent for EEG registration. Oral informed consent was obtained at follow up.

\subsection{Patients}

Consecutive, adult, comatose patients after cardiac arrest (Glasgow Coma Scale score $\leq 8$ ), admitted to the ICU, were included in our prospective cohort study. Exclusion criteria were concomitant acute stroke, traumatic brain injury, hanging/choking, auto intoxication, anaphylactic shock, drowning, preexisting dependency, severe spinal cord injury, or progressive neurodegenerative disease.

\subsection{Treatment}

"Patients were treated according to standard protocols for comatose patients after cardiac arrest. This included targeted temperature management at $33^{\circ} \mathrm{C}$ or $36^{\circ} \mathrm{C}$. In Rijnstate Hospital, since February 2014 , the target temperature was set from $33^{\circ} \mathrm{C}$ to $36^{\circ} \mathrm{C}$. In Medisch Spectrum Twente, there was a gradual shift from $33^{\circ} \mathrm{C}$ to $36^{\circ} \mathrm{C}$ in 2017 . Target temperature was induced as soon as possible after arrival at the emergency room or ICU and maintained for $24 \mathrm{~h}$. After $24 \mathrm{~h}$, passive re-warming was controlled to a speed of $0.25^{\circ} \mathrm{C}$ or $0.5^{\circ} \mathrm{C}$ per hour. In case of $T>38^{\circ} \mathrm{C}$ and a Glascow Coma Scale score $\leq 8$, targeted temperature management was restarted at $36.5-37.5^{\circ} \mathrm{C}$ for another $48 \mathrm{~h}$. In Medisch Spectrum
Twente, propofol and fentanyl or remifentanil were used for sedation. In Rijnstate Hospital, patients received a combination of propofol, midazolam, and/or morphine. Mostly, analgo-sedation was discontinued at a body temperature of $36.5^{\circ} \mathrm{C}$. In both hospitals, a non-depolarizing muscle relaxant (rocuronium or atracurium) was occasionally added in case of severe compensatory shivering." (Hofmeijer et al., 2015).

\subsection{Decisions on withdrawal of treatment}

"Withdrawal of treatment was considered $\geq 72 \mathrm{~h}$ after cardiac arrest, during normothermia, and off sedation. Decisions on treatment withdrawal were based on international guidelines including bilateral absence of evoked SSEPs, absent or extensor motor responses, incomplete return of brainstem reflexes and treatment resistant myoclonus (Sandroni et al., 2014; Wijdicks et al., 2006). Discontinuation of life sustaining treatment was sporadically initiated between $48 \mathrm{~h}$ and $72 \mathrm{~h}$ in case of absent SSEP responses. EEG data were not used for decisions regarding treatment withdrawal. However, physicians were not blinded to the EEG and treatment of electrographic seizures was left to the discretion of the treating physician." (Ruijter et al., 2018).

\subsection{Outcome}

"The primary outcome measure was neurological outcome expressed as the score on the five-point Glasgow-Pittsburgh Cerebral Performance Category (CPC) at six months (Cummins et al., 1991). Outcome was dichotomized as "good" (CPC 1-2) or "poor" (CPC 3-5). CPC scores were obtained by telephone follow-up at 6 months by one of the investigators (MTC, BR, or HK), blinded for EEG patterns and SSEP recordings. Scoring was based on a Dutch translation of the EuroQol-6D questionnaire." (Hofmeijer et al., 2015).

\subsection{EEG registrations and analyses}

“Continuous EEG registrations were started as soon as possible in all patients after arrival at the ICU. Twenty-one silversilverchloride cup electrodes were placed on the scalp according to the international $10-20$ system. A Neurocenter EEG recording system (Clinical Science Systems, Leiden, The Netherlands) or a Nihon Kohden system (VCM Medical, Leusden, The Netherlands) were used to make EEG registrations.

All EEG analyses were prespecified and performed offline, after the registrations. Epochs of 5 min were automatically selected by a computer algorithm at $12,24,48$, and $72 \mathrm{~h}$ after cardiac arrest (Tjepkema-Cloostermans et al., 2013)" (Hofmeijer et al., 2015). Additionally, five-minute epochs were selected at the time of SSEP recording $\pm 10 \mathrm{~h}$, preferably within $1 \mathrm{~h}$ from the SSEP recording.

Anonymized epochs with raw EEG data were presented to a reviewer (MvP, JH, BR, MTC, HK or AG) by the computer, in random order, blinded to the point in time of the epoch, the patient's clinical status during the recording, medication, and outcome. EEG epochs were visually classified by two reviewers, independently. "Upon disagreement, consensus was determined. If necessary, a third reviewer was consulted" (Hofmeijer et al., 2015).

Epochs were classified as suppressed $(<10 \mu \mathrm{V})$, low-voltage $(<20 \mu \mathrm{V})$, synchronous patterns on suppressed background ( $\geq 50 \%$ ) (burst suppression with identical bursts, generalized periodic discharges (GPD) on a suppressed background, or burstsuppression with generalized abrupt onset bursts with suppressed background), other burst-suppression patterns, GPDs with other background, other epileptiform patterns and continuous patterns (dominant frequencies $<4 \mathrm{~Hz}, 4-8 \mathrm{~Hz},>8 \mathrm{~Hz}$ ) (Hofmeijer et al., 2014; Ruijter et al., 2019a, 2015). “Burst-suppression” was defined 
as clear increases in amplitude (bursts) with interburst intervals of at least one second with low-voltage or absent activity (suppressions, $<10 \mu \mathrm{V}$ ) (Hofmeijer et al., 2015). Subsequently, burst suppression with identical bursts (Hofmeijer et al., 2014) and highly epileptiform bursts typically meet the criteria of "synchronous burst-suppression" (Ruijter et al., 2019a). GPD patterns were subdivided into GPDs on a suppressed background and GPDs with other background activity (Ruijter et al., 2015). Continuous patterns were subdivided according to their dominant frequency (delta, theta, or $\geq$ alpha).

\subsection{SSEP recordings and analyses}

In the first 55 patients, repeated SSEP recordings during the first 5 days of the ICU stay, or until discharge from the ICU, were intended. For later patients, SSEP recordings were done at the discretion of the treating physician. Generally, SSEPs were recorded between 48 and $72 \mathrm{~h}$ in patients who remained comatose after restoration of normothermia, off-sedation. "The SSEP was measured after stimulation of the right and left median nerve using a bipolar surface electrode at the wrist. Stimulus duration was set at $0.3 \mathrm{~ms}$ and stimulus amplitude was adjusted until a visible twitch of the thumb was produced. Two sets of $>200-1000$ responses were averaged, band pass filtered between $0.1 \mathrm{~Hz}$ and $2.5 \mathrm{kHz}$, and notch filtered around $50 \mathrm{~Hz}$. Stimulus frequency was set at 1.7 or $2.3 \mathrm{~Hz}$. Silver-silver chloride cup electrodes were placed at Erb's point, cervical spine (C5), and $2 \mathrm{~cm}$ posterior to C3 and $\mathrm{C} 4$ ( $\mathrm{C}^{\prime}$ and $\left.\mathrm{C4} 4^{\prime}\right)$. Fz was used as a reference." (Cloostermans et al., 2012). Peripheral N9 and N13 responses, and cortical N20 responses were recorded. SSEP results were categorized as present or absent based on the presence or absence of $\mathrm{N} 20$ responses. Unilateral present N20 responses were considered as present SSEP. SSEP recordings were classified as inconclusive if noise level was higher than signal or in the absence of peripheral responses. SSEP recordings were made using a Nicolet Bravo system (Viasys, Houten, The Netherlands).

\subsection{Statistical analyses}

Demographic, baseline, EEG, and SSEP data are presented in a descriptive way. Differences between groups of patients were tested by a chi-square test for categorical variables and Student's $t$-test for continuous variables, given a normal distribution. Sensitivity, specificity, positive predictive value (PPV), and negative predictive value (NPV) were calculated for (groups of) predictors of poor or good outcome, including corresponding 95\% confidence intervals. Association between SSEP and EEG is expressed as risk ratio (RR). Interobserver agreement of the EEG classification was analyzed with Cohen's Kappa. Analysis was performed with SPSS-22 (IBM).

\subsection{Data availability statement}

Raw EEG and outcome data used for this article will be kept for at least 15 years and made available upon request, for verification of results or new relevant research questions, conditionally. Conditions include optimal data safety, adequate methodology, mutual appointments on collaboration, and approval of all collaborators.

\section{Results}

EEG registrations were started in 647 patients. Twenty-eight patients were excluded from this analysis because they met one of the exclusion criteria: relevant traumatic brain injury, hanging/choking, preexisting dependency, auto intoxication, anaphylactic shock, drowning, recent ischemic stroke, severe spinal cord injury, or progressive neurodegenerative disease (Fig. 1). 619 consecutive patients were included, 352 in MST and 267 in Rijnstate Hospital. Baseline characteristics are summarized in Table 1 . Seventeen subjects (2.7\%) were lost to follow up, leaving 602 inclusions for analysis (Fig. 1). Of 14 patients, the CPC score at three months was used. Poor neurological outcome occurred in 314 patients (52\%), of whom 287 died. The EEG pattern could be classified in 356 patients at $12 \mathrm{~h}$, in 525 patients at $24 \mathrm{~h}$, in 398 patients at $48 \mathrm{~h}$ and in 241 patients at $72 \mathrm{~h}$. EEG classification was missing in case of abundant artifacts, at $12 \mathrm{~h}$ if EEG was started later than $12 \mathrm{~h}$ after cardiac arrest, and at $\geq 24 \mathrm{~h}$ if the patient had already died or woken up.

\subsection{Prediction of poor outcome}

Description of EEG and SSEP findings: At 12, 24, 48, and $72 \mathrm{~h}$ after cardiac arrest, a suppressed or synchronous pattern on suppressed background was observed in $87,84,36$, and 16 patients,

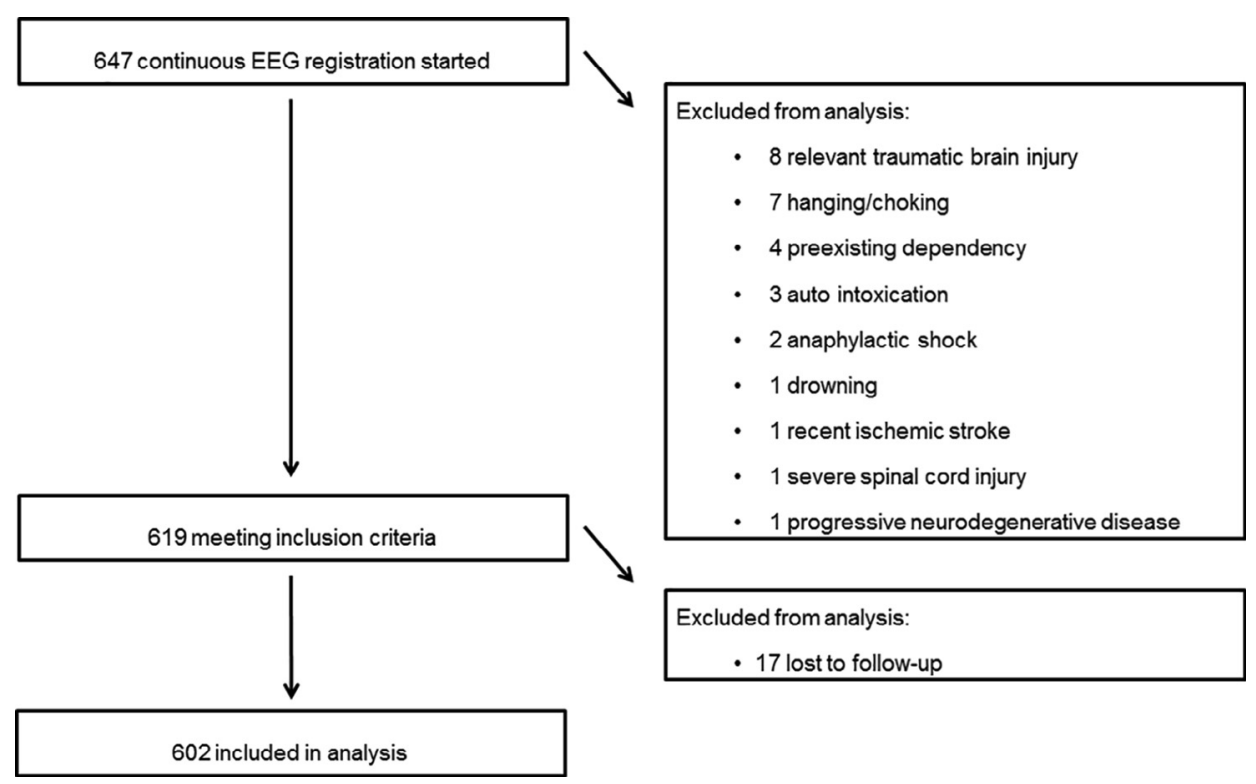

Fig. 1. Flow of patients through this study. 
Table 1

Patient characteristics and differences between patients with good and poor neurological outcome.

\begin{tabular}{|c|c|c|c|}
\hline & $\begin{array}{l}\text { Good outcome } \\
N=288\end{array}$ & $\begin{array}{l}\text { Poor outcome } \\
N=314\end{array}$ & $p$-value \\
\hline Female sex & $68(24 \%)$ & $74(24 \%)$ & 1 \\
\hline Mean age $( \pm S D)$ & $59 \pm 12$ & $66 \pm 12$ & $<0.001$ \\
\hline $\mathrm{OHCA}$ & $265 / 288(92 \%)$ & $279 / 314(89 \%)$ & 0.1 \\
\hline Cardiac etiology & $256 / 269(95 \%)$ & $228 / 284(80 \%)$ & $<0.001$ \\
\hline VF rhythm & $254 / 288(88 \%)$ & $176 / 314(56 \%)$ & $<0.001$ \\
\hline Mild therapeutic hypothermia $\left(33^{\circ} \mathrm{C}\right)$ & $172 / 240(72 \%)$ & $201 / 263(76 \%)$ & 0.2 \\
\hline Propofol in first $24 \mathrm{~h}$ & $269 / 281(96 \%)$ & $274 / 305(90 \%)$ & 0.005 \\
\hline Max propofol dose in first $24 \mathrm{~h}(\mathrm{mg} / \mathrm{kg} / \mathrm{h})$ & $2.93 \pm 1.16$ & $2.63 \pm 1.03$ & 0.002 \\
\hline Midazolam in first $24 \mathrm{~h}$ & $89 / 281(32 \%)$ & $108 / 305(35 \%)$ & 0.2 \\
\hline Max midazolam dose in first $24 \mathrm{~h}(\mu \mathrm{g} / \mathrm{kg} / \mathrm{h})$ & $117.7 \pm 73.0$ & $129.8 \pm 92.7$ & 0.3 \\
\hline Suppressed or synchronized EEG with suppressed background EEG at $12 \mathrm{~h}$ & $0 / 181(0 \%)$ & $87 / 175(50 \%)$ & $<0.001$ \\
\hline Suppressed or synchronized EEG with suppressed background at $24 \mathrm{~h}$ & $0 / 274(0 \%)$ & $84 / 251(34 \%)$ & $<0.001$ \\
\hline Suppressed or synchronized EEG with suppressed background at $48 \mathrm{~h}$ & $0 / 191(0 \%)$ & $36 / 207(17 \%)$ & $<0.001$ \\
\hline Suppressed or synchronized EEG with suppressed background at $72 \mathrm{~h}$ & $0 / 108(0 \%)$ & $16 / 133(12 \%)$ & $<0.001$ \\
\hline Bilaterally absent SSEP & $0 / 40(0 \%)$ & $87 / 204(43 \%)$ & $<0.001$ \\
\hline Continuous EEG at $12 \mathrm{~h}$ & $83 / 181(46 \%)$ & $17 / 175(10 \%)$ & $<0.001$ \\
\hline
\end{tabular}

$\mathrm{SD}=$ standard deviation; $\mathrm{OHCA}=$ out of hospital cardiac arrest; $\mathrm{VF}$ = ventricular fibrillation; SSEP = somatosensory evoked potential.

Table 2

Predictive values of (combinations of) EEG and SSEP parameters.

\begin{tabular}{|c|c|c|c|c|c|}
\hline Predictor & Predicted outcome & $\begin{array}{l}\text { Specificity } \\
(95 \% \text { CI })\end{array}$ & $\begin{array}{l}\text { Sensitivity } \\
(95 \% \mathrm{CI})\end{array}$ & $\begin{array}{l}\text { PPV } \\
(95 \% \mathrm{CI})\end{array}$ & $\begin{array}{l}\text { NPV } \\
(95 \% \mathrm{CI})\end{array}$ \\
\hline Continuous EEG pattern at $12 \mathrm{~h}$ & Good & $\begin{array}{l}90 \% \\
(84-94)\end{array}$ & $\begin{array}{l}46 \% \\
(38-53)\end{array}$ & $\begin{array}{l}83 \% \\
(74-90)\end{array}$ & $\begin{array}{l}62 \% \\
(55-68)\end{array}$ \\
\hline Suppressed or synchronized EEG with suppressed background at $24 \mathrm{~h}$ & Poor & $\begin{array}{l}100 \% \\
(98-100)\end{array}$ & $\begin{array}{l}27 \% \\
(22-32)\end{array}$ & $\begin{array}{l}100 \% \\
(95-100)\end{array}$ & $\begin{array}{l}56 \% \\
(51-60)\end{array}$ \\
\hline Absent SSEP, typically $48-72 \mathrm{~h}$ after resuscitation & Poor & $\begin{array}{l}100 \% \\
(98-100)\end{array}$ & $\begin{array}{l}28 \% \\
(23-33)\end{array}$ & $\begin{array}{l}100 \% \\
(95-100)\end{array}$ & $\begin{array}{l}56 \% \\
(52-60)\end{array}$ \\
\hline Suppressed or synchronized EEG with suppressed background at $24 \mathrm{~h}$ or absent SSEP & Poor & $\begin{array}{l}100 \% \\
(98-100)\end{array}$ & $\begin{array}{l}39 \% \\
(33-44)\end{array}$ & $\begin{array}{l}100 \% \\
(96-100)\end{array}$ & $\begin{array}{l}60 \% \\
(55-64)\end{array}$ \\
\hline
\end{tabular}

$\mathrm{CI}=$ confidence interval; SSEP $=$ somatosensory evoked potential.

and invariably associated with a poor outcome. Bilaterally absent SSEP responses were observed in 87 patients and also invariably associated with a poor outcome. Fifty patients with a poor outcome had a suppressed or synchronized EEG on suppressed background at $24 \mathrm{~h}$ after cardiac arrest and an absent SSEP.

Predictive values: Sensitivity, specificity, PPV and NPV of (a combination of) EEG and SSEP factors associated with a poor outcome are summarized in Table 2. Thirty-four patients with a poor outcome could be identified based on EEG pattern (suppressed or synchronous pattern on suppressed background) at $24 \mathrm{~h}$ after cardiac arrest, but had a present SSEP response. Thirty-seven patients with a poor outcome had an absent SSEP, but continuous or other EEG pattern at $24 \mathrm{~h}$ after cardiac arrest.

\subsection{Prediction of good outcome}

Description of EEG and SSEP findings: Continuous EEG patterns at $12 \mathrm{~h}$ after cardiac arrest were observed in 83 patients with a good outcome vs. 17 with a poor outcome. Predictive values: Specificity, sensitivity, PPV and NPV are summarized in Table 2. Beyond $12 \mathrm{~h}$, the observed incidence of continuous patterns among patients with a poor outcome grew. Consequently, the specificity of continuous patterns for prediction of good outcome decreased after $12 \mathrm{~h}$ (data not shown).

\subsection{Repeated SSEP}

In 55 patients, daily SSEP recordings during the first five days after cardiac arrest were planned. All were treated with mild therapeutic hypothermia. One of these patients had no SSEP recording and seven had only one, because of death within $32 \mathrm{~h}$ after cardiac arrest. Eight patients had two SSEP recordings, fourteen patients had three SSEP recordings on day 1-3, eleven patients had four SSEP recordings on day $1-4$, fifteen had five SSEP recordings on day $1-5$. In one patient, the first SSEP was considered inconclusive, whereas it was present on the following recording. One patient had present responses in the first four recordings and absent responses in the fifth SSEP recording. In all other patients with repeated SSEP recordings, N20 responses were either present $(n=40)$, or absent in all recordings $(n=5)$.

\subsection{Association of SSEP and EEG pattern}

A total of 360 SSEP recordings were done in 244 patients. Three hundred twenty-six of the according EEG epochs (91\%) were obtained at the time of SSEP recording $\pm 1 \mathrm{~h}$. The remaining epochs were collected at a mean of $3 \mathrm{~h}$ before or after SSEP recording. SSEP results in relation to the EEG background pattern at the moment of the SSEP recording are summarized in Table 3 . All patients with continuous, normal voltage $(>20 \mu \mathrm{V})$ EEG patterns with a dominant frequency $>8 \mathrm{~Hz}$ had preserved $\mathrm{N} 20$ responses. However, none of the other EEG patterns was invariably associated with a preserved $\mathrm{N} 20$ response, and none of the EEG patterns was invariably associated with an absent N20 response. If EEG and SSEP results were stratified to time since cardiac arrest, these results were essentially the same (see Appendix A: supplementary material).

\subsection{Interobserver agreement}

Interobserver agreement for designation of a suppressed or synchronized EEG on suppressed background was $0.72-0.80$, depending on the timing of the EEG classification. 
Table 3

SSEP results per EEG pattern.

\begin{tabular}{|c|c|c|c|c|c|}
\hline EEG pattern & $\begin{array}{l}\text { N20 not bilaterally absent } \\
N=247\end{array}$ & $\begin{array}{l}\text { N20 bilaterally absent } \\
N=92\end{array}$ & $P$-value & $\mathrm{RR}$ & $95 \% \mathrm{CI}$ \\
\hline Suppressed $(A \leq 10 \mu \mathrm{V})$ & $2(1 \%)$ & $6(6 \%)$ & 0.007 & 2.9 & $1.9-4.5$ \\
\hline Low-voltage $(10 \mu \mathrm{V}<A \leq 20 \mu \mathrm{V})$ & $12(5 \%)$ & $11(11 \%)$ & 0.04 & 1.9 & $1.2-3.0$ \\
\hline Burst suppression with identical bursts & $4(2 \%)$ & $8(8 \%)$ & 0.005 & 2.6 & $1.7-4.0$ \\
\hline $\begin{array}{l}\text { Burst-suppression with generalized, abrupt onset bursts, } \\
\text { with suppressed background }\end{array}$ & $1(<1 \%)$ & $5(5 \%)$ & 0.008 & 3.2 & $2.1-4.8$ \\
\hline Other burst suppression & $82(32 \%)$ & $19(20 \%)$ & 0.03 & 0.6 & $0.4-1.0$ \\
\hline Continuous $<4 \mathrm{~Hz}$ & $33(13 \%)$ & $7(7 \%)$ & 0.2 & 0.6 & $0.3-1.2$ \\
\hline Continuous $4-8 \mathrm{~Hz}$ & $55(22 \%)$ & $11(11 \%)$ & 0.05 & 0.6 & $0.3-1.0$ \\
\hline Continuous $>8 \mathrm{~Hz}$ & $20(8 \%)$ & $0(0 \%)$ & 0.01 & 0.0 & $0.0-0.0$ \\
\hline GPDs with suppressed background & $13(5 \%)$ & $15(15 \%)$ & 0.002 & 2.2 & $1.5-3.2$ \\
\hline GPDs with other background & 22 (9\%) & $8(8 \%)$ & 0.9 & 1.0 & $0.5-1.8$ \\
\hline Other epileptiform patterns & $3(1 \%)$ & $2(2 \%)$ & 0.9 & 1.5 & $0.5-4.4$ \\
\hline
\end{tabular}

SSEP = somatosensory evoked potential; RR = Risk Ratio for absent SSEP; CI = confidence interval; GPD = generalized periodic discharges .

\section{Discussion}

We confirm that absent SSEP and suppressed or synchronized EEG on suppressed background are reliable predictors of poor outcome of comatose patients after cardiac arrest, and complementary with regard to identification of patients with a poor outcome. Furthermore, for the first time, we demonstrate that the EEG background pattern at the time of SSEP testing does not reliably predict presence or absence of the SSEP response. This is with the exception of continuous, normal voltage traces with a $>8 \mathrm{~Hz}$ frequency content, where N20 responses were always preserved. There was no relevant additional value of daily SSEP testing over single SSEP testing, since SSEP responses were either present or absent every day in the vast majority of patients.

Complementarity of SSEP and EEG for prediction of poor outcome is in line with previous publications on outcome prediction after cardiac arrest using multimodal approaches (Cloostermans et al., 2012; Grippo et al., 2017; Hofmeijer et al., 2015; Nolan et al., 2015; Oddo and Rossetti, 2014; Sivaraju et al., 2015; Tsetsou et al., 2018). Apparently, SSEP and EEG partly identify different patients with severe encephalopathy and a subsequent poor outcome. Continuous EEG patterns at $12 \mathrm{~h}$ after cardiac arrest are strongly associated with a good neurological recovery (Hofmeijer et al., 2015; Tjepkema-Cloostermans et al., 2015). However, preserved SSEP responses are not specific for good outcome (Zandbergen et al., 2006). In these patients, the EEG may differentiate between patients with good and poor prognosis (Rossetti et al., 2009; Rothstein et al., 2010). Specific SSEP characteristics, such as N20 amplitude $<0.62 \mu \mathrm{V}$, may contribute to prediction of poor neurological outcome (Endisch et al., 2015), but have not been studied with regard to prediction of good outcome.

To further study the association between SSEP and EEG, we analyzed the N20 response in relation to the EEG pattern at the time of SSEP testing. Suppressed EEG and synchronous patterns on suppressed background were associated with absent SSEPs, but not invariably so. Even with suppressed or synchronous EEG on a suppressed background, N20 responses could be evoked in some patients. Otherwise, with continuous, traces, SSEPs could be absent.

The origin of pathological EEG and SSEP traces cannot be derived from the data. Previous studies to clarify mechanisms used divergent experimental models. Still, the pathophysiology of (pathological) EEG patterns is only partly known and many explanations are, at least partly, speculative. In a previous mathematical study we hypothesized that somatosensory evoked responses, which reflect extracellular currents generated by excitatory postsynaptic potentials, depend on a preserved thalamocortical synaptic transmission to somatosensory cortical neurons (van Putten,
2012). Otherwise, some pathological EEG patterns that we typically observe after cardiac arrest seemed to be associated with disturbed synaptic transmission between pyramidal cells (Ruijter et al., 2017; Tjepkema-Cloostermans et al., 2014). In a postmortem study, where EEG and SSEP findings were related to results from histological analyses from the brains of non-survivors after cardiac arrest, we observed that absent SSEP responses were always accompanied with severe thalamic damage. Burst-suppression patterns with identical bursts were associated with damage to cortex and cerebellum (van Putten et al., 2019). Others have shown that diffuse slowing of the EEG may be associated with disturbed thalamocortical interactions, which in turn may result from subcortical or cortical ischemic damage (Guillery, 1995; van Wijngaarden et al., 2016). Apparently, hypoxic-ischemic brain damage may selectively affect particular systems or networks in the brain, each leading to particular EEG or SSEP patterns. However, details remain unknown.

For prediction of outcome, we followed previous reports and analyzed SSEP at $3 \pm 1$ days after cardiac arrest and EEG within the first $24 \mathrm{~h}$. This indicates that these SSEPs were generally obtained at normothermia and off sedation, whereas EEG was analyzed during treatment with targeted temperature management and sedation. Studies relating absent N20 responses to poor outcome report on a small number of false positives, if SSEP testing was done during hypothermia and sedation (Sandroni et al., 2014). Therefore, guidelines recommend to use SSEP at normothermia (Sandroni et al., 2014). Otherwise, with EEG, the largest differences between patients with good and poor outcome are observed within $24 \mathrm{~h}$ and predictive values are high despite hypothermia and sedation (Tjepkema-Cloostermans et al., 2015, 2013). Patients precluding cerebral recovery display most specific patterns predicting poor outcome within the first $24 \mathrm{~h}$ after cardiac arrest. In later phases, the EEG tends to change to less specific patterns (Hofmeijer et al., 2015). Although the EEG during treatment with sedative drugs or hypothermia is generally deemed as " not interpretable" we emphasize that suppressed, and synchronous patterns with suppressed background cannot be induced solely by midazolam or propofol in the dosages that are generally used in the ICU. This assumption is supported by recent research (Ruijter et al., 2019b).

Our study has certain limitations. First, as in almost all unblinded studies on diagnostic accuracy the self-fulling prophecy is a potential problem. In this study, international guidelines on treatment continuation were strictly followed. Life sustaining treatment was mostly interrupted with an absent SSEP response. Otherwise, the EEG was not used for decisions on discontinuation of life sustaining treatment. Second, although EEG registrations were performed in all patients, SSEP was only done systematically 
in the first 55 patients that were included. In later patients, SSEP was done on indication, which indicates that SSEPs were tested in the subgroup of patients with sustained unresponsiveness after weaning from targeted temperature management, introducing selection bias. Third, both SSEP and EEG were visually analyzed, which is subjective to personal preferences. While EEGs were classified off line, blinded for patients' clinical status, treatment, and outcome, SSEP classification was done at the bedside.

\section{Conclusion}

SSEP and EEG are complementary for prediction of poor outcome of comatose patients after cardiac arrest. SSEP response cannot be predicted from the EEG pattern. Differences between SSEP and EEG patterns probably reflect selective damage of either thalamocortical or inter-pyramidal synapses.

\section{Study funding}

This project is funded by the Dutch Ministry of Economic Affairs, Agriculture and Innovation, province Overijssel and Gelderland (ViP Brain Networks project).

HMK is financially supported by the Rijnstate-Radboudumc promotion fund.

BJR was financially supported by the Dutch Epilepsy Fund (Epilepsiefonds, grant reference NEF 14-18).

\section{Declaration of Competing Interest}

M.J.A.M. van Putten is co-founder of Clinical Science Systems, which is a supplier of EEG systems for one of the participating sites (Medisch Spectrum Twente). Clinical Science Systems did not provide funding and was not involved in the design, execution, analysis, interpretation or publication of the study. The other authors do not report any conflicts of interest.

\section{Appendix A. Supplementary material}

Supplementary data to this article can be found online at https://doi.org/10.1016/j.clinph.2019.08.022.

\section{References}

Buzsaki G, Anastassiou CA, Koch C. The origin of extracellular fields and currentsEEG, ECoG, LFP and spikes. Nat Rev Neurosci 2012;13(6):407-20.

Cloostermans MC, van Meulen FB, Eertman CJ, Hom HW, van Putten MJ. Continuous electroencephalography monitoring for early prediction of neurological outcome in postanoxic patients after cardiac arrest: a prospective cohort study. Critical Care Med 2012;40(10):2867-75.

Cummins RO, Chamberlain DA, Abramson NS, Allen M, Baskett PJ, Becker L, et al. Recommended guidelines for uniform reporting of data from out-of-hospital cardiac arrest: the Utstein Style. A statement for health professionals from a task force of the American Heart Association, the European Resuscitation Council, the Heart and Stroke Foundation of Canada, and the Australian Resuscitation Council. Circulation 1991;84(2):960-75.

Endisch C, Storm C, Ploner CJ, Leithner C. Amplitudes of SSEP and outcome in cardiac arrest survivors: a prospective cohort study. Neurology 2015;85 (20):1752-60.

Grippo A, Carrai R, Scarpino M, Spalletti M, Lanzo G, Cossu C, et al. Neurophysiological prediction of neurological good and poor outcome in post-anoxic coma. Acta Neurol Scand 2017;135(6):641-8.

Guillery RW. Anatomical evidence concerning the role of the thalamus in corticocortical communication: a brief review. J Anatomy 1995;187:583-92.

Hofmeijer J, Beernink TM, Bosch FH, Beishuizen A, Tjepkema-Cloostermans MC, van Putten MJ. Early EEG contributes to multimodal outcome prediction of postanoxic coma. Neurology 2015;85(2):137-43.

Hofmeijer J, Tjepkema-Cloostermans MC, van Putten MJ. Burst-suppression with identical bursts: a distinct EEG pattern with poor outcome in postanoxic coma. Clin Neurophysiol 2014;125(5):947-54.
Nolan JP, Soar J, Cariou A, Cronberg T, Moulaert VRM, Deakin CD, et al. European Resuscitation Council and European Society of Intensive Care Medicine 2015 guidelines for post-resuscitation care. Intensive Care Med 2015;41:2039-56.

Oddo M, Rossetti AO. Early multimodal outcome prediction after cardiac arrest in patients treated with hypothermia. Critical Care Med 2014;42(6):1340-7.

Oh SH, Park KN, Shon YM, Kim YM, Kim HJ, Youn CS, et al. Continuous amplitudeintegrated electroencephalographic monitoring is a useful prognostic tool for hypothermia-treated cardiac arrest patients. Circulation 2015;132 (12):1094-103.

Rossetti AO, Oddo M, Liaudet L, Kaplan PW. Predictors of awakening from postanoxic status epilepticus after therapeutic hypothermia. Neurology 2009;72(8):744-9.

Rothstein T. Does hypothermia influence the predictive value of bilateral absent N20 after cardiac arrest? Neurology 2010;75(6):575-6. author reply -6.

Ruijter BJ, Hofmeijer J, Meijer HGE, van Putten M. Synaptic damage underlies EEG abnormalities in postanoxic encephalopathy: a computational study. Clin Neurophysiol 2017;128(9):1682-95.

Ruijter BJ, Hofmeijer J, Tjepkema-Cloostermans MC, van Putten M. The prognostic value of discontinuous EEG patterns in postanoxic coma. Clin Neurophysiol 2018;129(8):1534-43.

Ruijter BJ, Tjepkema-Cloostermans MC, Tromp SC, van den Bergh WM, Foudraine NA, Kornips FHM, et al. Early EEG for outcome prediction of postanoxic coma: a prospective cohort study. Ann Neurol 2019.

Ruijter BJ, van Putten M, van den Bergh WM, Tromp SC, Hofmeijer J. Propofol does not affect the reliability of early EEG for outcome prediction of comatose patients after cardiac arrest. Clin Neurophysiol 2019b;130(8):1263-70.

Ruijter BJ, van Putten MJ, Hofmeijer J. Generalized epileptiform discharges in postanoxic encephalopathy: quantitative characterization in relation to outcome. Epilepsia 2015;56(11):1845-54.

Sandroni C, Cariou A, Cavallaro F, Cronberg T, Friberg H, Hoedemaekers C, et al. Prognostication in comatose survivors of cardiac arrest: an advisory statement from the European Resuscitation Council and the European Society of Intensive Care Medicine. Resuscitation 2014;85(12):1779-89.

Sivaraju A, Gilmore EJ, Wira CR, Stevens A, Rampal N, Moeller JJ, et al. Prognostication of post-cardiac arrest coma: early clinical and electroencephalographic predictors of outcome. Intensive Care Med 2015;41 (7):1264-72.

Sondag L, Ruijter BJ, Tjepkema-Cloostermans MC, Beishuizen A, Bosch FH, van Til JA, et al. Early EEG for outcome prediction of postanoxic coma: prospective cohort study with cost-minimization analysis. Critical Care 2017;21(1):111.

Spalletti M, Carrai R, Scarpino M, Cossu C, Ammannati A, Ciapetti M, et al. Single electroencephalographic patterns as specific and time-dependent indicators of good and poor outcome after cardiac arrest. Clin Neurophysiol 2016;127 (7):2610-7.

Tjepkema-Cloostermans MC, Hindriks R, Hofmeijer J, van Putten MJ. Generalized periodic discharges after acute cerebral ischemia: reflection of selective synaptic failure? Clin Neurophysiol 2014;125(2):255-62.

Tjepkema-Cloostermans MC, Hofmeijer J, Beishuizen A, Hom HW, Blans MJ, Bosch $\mathrm{FH}$, et al. Cerebral recovery index: reliable help for prediction of neurologic outcome after cardiac arrest. Critical Care Med 2017;45(8):e789-97.

Tjepkema-Cloostermans MC, Hofmeijer J, Trof RJ, Blans MJ, Beishuizen A, van Putten MJ. Electroencephalogram predicts outcome in patients with postanoxic coma during mild therapeutic hypothermia. Critical Care Med 2015;43(1):159-67.

Tjepkema-Cloostermans MC, van Meulen FB, Meinsma G, van Putten MJ. A Cerebral Recovery Index (CRI) for early prognosis in patients after cardiac arrest. Critical Care 2013;17(5):R252.

Tsetsou S, Novy J, Pfeiffer C, Oddo M, Rossetti AO. Multimodal outcome prognostication after cardiac arrest and targeted temperature management: analysis at $36{ }^{\circ} \mathrm{C}$. Neurocritical Care 2018;28(1):104-9.

van Putten M, Jansen C, Tjepkema-Cloostermans MC, Beernink TMJ, Koot R, Bosch F, et al. Postmortem histopathology of electroencephalography and evoked potentials in postanoxic coma. Resuscitation 2019;134:26-32.

van Putten MJ. The N20 in post-anoxic coma: are you listening? Clin Neurophysiol 2012;123(7):1460-4.

van Wijngaarden JB, Zucca R, Finnigan S, Verschure PF. The impact of cortical lesions on thalamo-cortical network dynamics after acute ischaemic stroke: a combined experimental and theoretical study. PLoS Comput Biol 2016;12(8): e1005048.

Wijdicks EF, Hijdra A, Young GB, Bassetti CL, Wiebe S. Quality Standards Subcommittee of the American Academy of N. Practice parameter: prediction of outcome in comatose survivors after cardiopulmonary resuscitation (an evidence-based review): report of the Quality Standards Subcommittee of the American Academy of Neurology. Neurology 2006;67(2):203-10.

Zandbergen EG, de Haan RJ, Stoutenbeek CP, Koelman JH, Hijdra A. Systematic review of early prediction of poor outcome in anoxic-ischaemic coma. Lancet 1998;352:1808-12.

Zandbergen EG, Hijdra A, Koelman JH, Hart AA, Vos PE, Verbeek MM, et al. Prediction of poor outcome within the first 3 days of postanoxic coma. Neurology 2006;66(1):62-8. 